\title{
A cohort study on Actinobacillus pleuropneumoniae colonisation in suckling piglets
}

\author{
T.J. Tobias ${ }^{\mathrm{a}, *}$, D. Klinkenberg ${ }^{\mathrm{a}}$, A. Bouma ${ }^{\mathrm{a}}$, J. van den Broek ${ }^{\mathrm{a}}$, A.J.J.M. Daemen ${ }^{\mathrm{a}}$, \\ J.A. Wagenaar ${ }^{\mathrm{b}, \mathrm{c}}$, J.A. Stegeman ${ }^{\mathrm{a}}$ \\ a Department of Farm Animal Health, Faculty of Veterinary Medicine, Utrecht University, Yalelaan 7, 3584 CL Utrecht, The Netherlands \\ b Department of Infectious Diseases and Immunology, Faculty of Veterinary Medicine, Utrecht University, Yalelaan 1, 3584 CL Utrecht, \\ The Netherlands \\ c Central Veterinary Institute of Wageningen UR, PO Box 65, 8200 AB Lelystad, The Netherlands
}

\section{A R T I C L E I N F O}

\section{Article history:}

Received 25 March 2013

Received in revised form 5 February 2014

Accepted 18 February 2014

\section{Keywords:}

Actinobacillus pleuropneumoniae

Pig-microbiological disease

Pigs

Pneumonia

Colonisation

Respiratory disease

\begin{abstract}
A B S T R A C T
Actinobacillus pleuropneumoniae causes respiratory disease in pigs and despite the use of preventive measures such as vaccination and antimicrobials clinical outbreaks still occur. At weaning often many piglets are not colonised. If differences in prevalence between litters are large and if factors were known that could explain these differences, this may provide an opportunity to raise groups of $A$. pleuropneumoniae free piglets. To this end, a cohort study was performed on two endemically infected farrow-to-finish farms. Seventy-six of 133 sows were selected using stratified random selection by parity. Farmers complied with a strict hygiene and animal management protocol to prevent transmission between litters. Tonsil brush and serum samples taken three weeks before parturition were tested for antigen with an apxIVA qPCR and antibodies with Apx and Omp ELISAs, respectively. Three days before weaning tonsil brush samples from all piglets $(n=871)$ were collected and tested for antigen. Whereas all sows tested positive both in serology tests as well as qPCR, 0.41 of the litters tested fully negative and 0.73 of all piglets tested negative. The proportion of positively tested piglets in positive litters ranged from $0.08-1.0$ (median $=0.36$ ). A grouped logistic regression model with a beta binomial distribution of the probability for piglets to become infected was fitted to the data and associations with explanatory variables were explored. To test the possibility that alternatively the clustering was caused by onwards transmission among the piglets, a transmission model was fitted to the data incorporating sow-piglet and piglet-piglet transmission, but this model did not fit better. The results of this study showed that the number of colonised suckling piglets was highly clustered and mainly attributable to the variability of infectiousness of the dam, but no dam related risk factor for colonisation status of litter or piglets within litters could be identified.
\end{abstract}

(C) 2014 Elsevier B.V. All rights reserved.

\section{Introduction}

One of the most common bacterial infections on pig farms is caused by Actinobacillus pleuropneumoniae.

\footnotetext{
* Corresponding author. Tel.: +31 30 2531248; fax: +31 302521887.

E-mail address: T.J.Tobias@uu.nl (T.J. Tobias).
}

Infections may often run a subclinical course, but can also result in clinical outbreaks, characterised by pleuritis, respiratory distress, and mortality (Gottschalk and Taylor, 2006). Control and prevention of clinical disease is currently achieved by use of antimicrobials (Mengelers et al., 2000; Hoflack et al., 2001), or vaccination (Tumamao et al., 2004). However, the use of antimicrobials does not clear A. pleuropneumoniae from tonsils of colonised pigs 
(Angen et al., 2008) and vaccination does not prevent colonisation of susceptible pigs (Velthuis et al., 2003). Therefore, these measures will not attribute to elimination of $A$. pleuropneumoniae from farms.

Piglets can already become colonised during the suckling stage (Vigre et al., 2002). However, despite the finding that the prevalence of $A$. pleuropneumoniae infections in sows was generally high (Chiers et al., 2002; Fablet et al., 2011; Sjölund et al., 2011), the prevalence of colonised piglets around the time of weaning was found to be rather low under controlled (Vigre et al., 2002) as well as under field conditions (Chiers et al., 2002). In these studies, no information was provided on the distribution of colonisation across litters. Clustering across litters may be expected if some dams transmit the infection more easily than others, and/or if the piglets transmit the bacterium amongst themselves. Understanding the factors that explain variation in colonisation could provide an opportunity to develop control measures on farms.

Differences between litters due to transmission by the dam could be expected if there is variation in maternally derived antibodies, which is related to the level of antibodies in the serum of the dam (Sjölund et al., 2011). It has been reported that these antibodies prevent the development of clinical signs (Cruijsen et al., 1995a), as disease is usually seen after the age at which maternal antibodies have waned (Cruijsen et al., 1995b). Whether antibodies protect pigs against colonisation of A. pleuropneumoniae is unknown, but convalescent serum of naturally infected pigs can inhibit bacterial cell adhesion to buccal epithelial cells in vitro (Hamer-Barrera et al., 2004). In turn, parity can affect the level of immunoglobulin passed on from dam to the piglets, but reports are contradictory on this matter. For example, Klobasa and Butler (1987) reported a positive association between parity and concentrations of immunoglobulin in milk, whereas Sjölund et al. (2011) found higher A. pleuropneumoniae antibody levels in serum of parity-one sows and Cruijsen et al. (1995b) reported higher antibody titres in offspring from younger sows. These observations raise the question whether characteristics of the dam (parity and antibody level) could be related to the probability that their piglets become colonised and whether sow characteristics can be identified as risk or protective factors.

The objectives of this study were to study clustering of colonisation status of suckling piglets just before weaning in endemically A. pleuropneumoniae infected pig farms, and to determine the association between dam parity, antibody levels and the probability of colonisation of litters and piglets within litters during the suckling period.

\section{Materials and methods}

A cohort study was performed on two farrow-to-finish farms (A and B) in two farrowing rooms (cohorts) per farm. Sows were examined for the presence of A. pleuropneumoniae infection by collection of blood and tonsil brush samples approximately three weeks before parturition. The proportions of colonisation at litter and individual piglet level were determined three days before weaning and associations with dam parity and sow serum and brush sample results were evaluated.

\subsection{Setting}

Two Dutch farrow-to-finish farms (farms A and B) were selected, both privately owned. The farms were selected for the following reasons. Both farms had a history of a high pleuritis and respiratory signs caused by A. pleuropneumoniae in finisher pigs. Furthermore, the farms had to raise their own breeding stock, had not used $A$. pleuropneumoniae vaccines in the year before the trial started and did not apply preventive antimicrobial batch treatment during suckling or after weaning. The farms were not related to each other. Finally, the willingness of the farmer and the ability on the farm to comply with the research protocol were the most important criteria for inclusion.

The study population consisted of a census sample of four cohorts with 76 sows and their offspring; 871 piglets. Each cohort was raised within one farrowing room. Samples from farm A were collected from April to June 2011 and samples from Farm B were collected from May to July 2012.

Farm A was a farrow-to-finish farm with 1700 sows; farm B housed 760 sows and $70 \%$ of the piglets were sold at 10 weeks of age, the rest was finished on site. Gestating sows in farm A were housed individually or in groups of five animals, whereas in farm $B$ gestating sows were housed in groups of approximately 54 sows. Confirmation of $A$. pleuropneumoniae infections was obtained by performing necropsy on diseased pigs (Gottschalk and Taylor, 2006) on farm A, and serology in growers and finishers on farm B. Additionally, from both farms an A. pleuropneumoniae serovar 2 isolate was recovered from affected lung tissue obtained at slaughterhouse investigations after the study period.

\subsection{Study population within farms}

In both farms, two farrowing week batches were selected based on the farms' schedules to be able to comply with the hygiene and management protocol. A total of 133 F1 sows, bred for finishing, were sampled three weeks before parturition and all tested positive in qPCR. From the four farrowing batches four study cohorts of sows were composed by computer aided stratified random selection. Stratification was performed on parity, relative to the parity distribution in the farrowing batch. On farm A, two cohorts of 18 sows were randomly selected from 38 and 26 sows originating from two farrowing week batches, two weeks apart, respectively. On farm B, two cohorts of 20 sows were selected from 36 and 33 sows, from two farrowing batches one week apart. Due to an expansion of farm B in 2009, relatively few parity-1 sows were present and all gilts from the farrowing week batch were selected for follow up. Subsequently 76 selected sows were randomly allocated to pens in the farrowing room. Finally 871 piglets were sampled.

For analysis sow parity was divided into two groups, based on the observed median; parity 1 and 2 (referred to as 'low') and parity 3 and higher, referred to as 
'high' (Supplementary Table 1). As on the farms in this study parity-1 sows were not treated differently (by vaccination or housing) and no signs of active infections were observed in parity- 1 sows, they were not considered separately as such for the data analysis like in other studies (Cruijsen et al., 1995b; Sjölund et al., 2011). Most importantly, the data did not show any evidence to treat the parity-1 sows differently in the analyses.

As both farms raise their own breeding stock, only F1 sows were selected that were bred for fattening and thereby all piglets were crossbreds. In this study, piglet sampling was planned at approximately 24 days of age, three days before weaning at approximately 27 days of age. The age of piglets at sampling varied, as sows farrowed on different days, which was thereby identified as a possible confounder. As the probability of colonisation was thought to increase with time, age of piglets was therefore included as an explanatory variable in statistical analyses. Neither farm workers, nor data collectors were informed about test results during the study to prevent observation or selection bias.

\subsection{Animal management and hygiene protocol}

As we aimed to study variation in colonisation within and between litters, contacts between litters were minimised. Moreover, original litters were kept intact as much as possible. Therefore, cross fostering was minimised: a maximum of four litters per cohort were permitted to receive piglets, not later than $24 \mathrm{~h}$ after birth. Any surplus piglets after applying cross fostering were moved to another room and were excluded from the study. Eventually it appeared that only a maximum of three litters per cohort received cross-fostered piglets. For these litters, cross-fostering was included as variable in the statistical analyses. During standard litter treatments (identification by ear number, iron injection and tail docking) and sample collection strict hygiene measures were taken. For each pen disposable gloves and boot covers and disposable needles were used.

\subsection{Sample collection and analysis}

Tonsil brush and serum samples were collected from sows approximately three weeks before parturition. The samples were analysed by ELISA for antibodies against the $42 \mathrm{kD}$ outer membrane protein (Omp) (Kobisch and van den Bosch, 1992), and ApxI, ApxII and ApxIII toxins (Nielsen et al., 2000) at MSD, Boxmeer, The Netherlands. Formal test characteristics for the tests are not available. Recently, a study that used recombinant produced toxin antigen, rather than purified toxins obtained from A. pleuropneumoniae, reported diagnostic sensitivities of 1.0, 0.95, 0.93 respectively and a diagnostic specificity of 0.96 for three Apx toxin ELISA tests (Shin et al., 2011). Antibody titres were expressed on a $\log _{2}$ scale, with a maximum of 14. For statistical analysis results were coded into high or low positive, based on the observed median result in the study.
Tonsil brush samples of sows and piglets were collected by brushing tonsils with a sterile toothbrush for $10 \mathrm{~s}$. Samples were processed and analysed with an apxIVA qPCR (with reported sensitivity and specificity of 0.98 and 1.0 respectively) (Tobias et al., 2012). To increase the rate of processing and reduce costs the sample handling protocol was slightly modified from the one described previously. Previously the sample was concentrated by immersion of the toothbrush in $10 \mathrm{~mL}$ saline for $20 \mathrm{~min}$, removal of the toothbrush, centrifugation, and suspension of the pellet in $0.5 \mathrm{~mL}$ saline and DNA extraction was performed with $0.2 \mathrm{~mL}$ of the $0.5 \mathrm{~mL}$ suspension (Tobias et al., 2012). In this study $1 \mathrm{~mL}$ of the $10 \mathrm{~mL}$ suspension was taken out for subsequent overnight storage and subsequent DNA isolation. After centrifugation of the $1 \mathrm{~mL}$ sample and discarding the supernatant, $0.2 \mathrm{~mL}$ of Instagene Matrix was added and the same protocol was applied as described previously. Finally $10 \mu \mathrm{L}$ of DNA was used for PCR analysis. Consequently, the minimal detection limit per sample was increased from 250 (as in Tobias et al., 2012) to 1000 colony forming units (CFUs). QPCR sample results were denoted as CFUs on a $\log _{10}$ scale, or as positive or negative.

\subsection{Ethics}

The samples were obtained in compliance with Dutch Law on Animal Experimentation and Act on the Practice of Veterinary Medicine. Informed consent was obtained from each participating farmer.

\subsection{Statistical analysis}

Dam qPCR results were compared between farms using Student's $t$-test and dam serum ELISA results by Wilcoxon rank sum test, because of the ordinal nature of the data. Correlations between dam related factors (antibody test results and parity) were analysed by the Spearman rank correlation.

Clustering of colonisation across litters and association between dam factors and piglet colonisation was addressed with a grouped logistic regression model on litter level (Supplementary files 1 and 2). In the full model, the number of positively tested piglets per litter was assumed to follow a zero-inflated beta-binomial distribution (see e.g. Cheung, 2006) to allow for two clustering mechanisms: the zero-inflation for the possibility that some sows would not be infectious at all, and the beta-binomial distribution which allows for overdispersion due to factors not explained by the independent variables (Agresti, 2013). The zero-inflation was modelled by parameter $p_{\text {dam }}$ (the probability for a dam to be infectious) and $\operatorname{logit}\left(p_{\text {dam }}\right)$ was allowed to depend linearly on the explanatory variables. The beta-binomial distribution was parameterised by the mean probability $p_{\text {piglet }}$ for a piglet (with an infectious dam) to be infected and a dispersion parameter $\rho$ which relate to the usual shape parameters as $p_{\text {piglet }}=\alpha /(\alpha+\beta)$ and $\rho=1 /(\alpha+\beta+1)$ (Agresti, 2013); the lower the value of $\rho$, the less overdispersion, with $\rho=0$ representing the binomial distribution. Logit $\left(p_{\text {piglet }}\right)$ was allowed to depend linearly on the explanatory variables. According to the model, the 
probability for the number of positive piglets $X$ in a litter of size $n$ is

$$
\begin{aligned}
& \operatorname{Pr}(X=0)=1-p_{\text {dam }}+p_{\text {dam }} \int_{0}^{1} \delta_{\beta\left(p_{\text {piglet }}, \rho\right)}(p) \delta_{\text {binom }(p, n)}(0) d p \\
& \operatorname{Pr}(X=x>0)=p_{\text {dam }} \int_{0}^{1} \delta_{\beta\left(p_{\text {piglet }}, \rho\right)}(p) \delta_{\text {binom }(p, n)}(x) d p
\end{aligned}
$$

in which $\delta_{\beta}(p)$ and $\delta_{\text {binom }}(x)$ are the densities of the beta and binomial distributions.

Explanatory variables (dam characteristics) were included either in both regression equations (for $p_{d a m}$ and $\left.p_{\text {piglet }}\right)$ at the same time, or in either probability separately. The absence of a zero-inflation $\left(p_{d a m}=1\right)$ and the absence of additional overdispersion ( $\rho=0$, i.e. a binomial distribution) were evaluated as special versions of the full model. This resulted in eight subgroups of models:

1. no zero-inflation and binomial distribution with explanatory variables

2. no zero-inflation and beta-binomial distribution with explanatory variables

3. zero-inflation with explanatory variables and binomial distribution without explanatory variables

4. zero-inflation with explanatory variables and betabinomial distribution without explanatory variables

5. zero-inflation without explanatory variables and binomial distribution with explanatory variables

6. zero-inflation without explanatory variables and betabinomial distribution with explanatory variables

7. zero-inflation and binomial distribution, both with explanatory variables

8. zero-inflation and beta-binomial distribution, both with explanatory variables

The nine dam characteristic explanatory variables that were evaluated in all combinations (cohort numbers 1-4, litters' age at sampling, dam parity, sow serology results (ApxI, ApxII, ApxIII, Omp), dam qPCR result and whether any litters were used for cross fostering) resulted in $2^{9}$ times 8 different models.

Each possible model was fitted by maximum likelihood estimation by numerical optimisation in $\mathrm{R}$ package bbmle (see supplementary material). For some models, several combinations of initial guesses had to be tried before finding the best fit, especially models of subtypes 3 and 4 if many explanatory variables were included.

Selection for the best model was performed based on AIC (Burnham and Anderson, 2002, p. 70). A lower AIC represents a better model and generally it is considered that models within two points of AIC are equivalent. Confidence intervals for model parameters were obtained by profile likelihood.

The best model contained no zero-inflation and a betabinomial distribution without explanatory variables (the simplest subgroup 2 model). Given the overdispersion as explained by the beta-binomial distribution, it is useful to know the difference in infection probability that could be identified with the current study design (for one explanatory variable in $\left.p_{\text {piglet }}\right)$. Therefore, we did a 'post hoc' power analysis. We simulated the infection outcome in two groups of 38 litters of 12 pigs ( 76 litters in total, as in our study), with beta-binomial distributions with $\rho=0.53$ as estimated, and a range of differences in $p_{\text {piglet }}$ between the two groups (the mean of which equal to 0.28 as estimated). For each difference in $p_{\text {piglet }}$ we analysed 1000 simulated datasets and estimated the power as the number of simulated datasets in which a difference was identified based on AIC (with a difference of two points). The $p_{\text {piglet }}$ for which the power was $80 \%$ was chosen as the difference that could have been found in our study.

Finally, a transmission model was fitted to the data as an alternative model to explain the observed clustering. In this model, it was assumed that the infection spreads within litters according to an SI-model: each piglet is infected by the sow at rate $\lambda_{\text {sow }}$ per day, and by the other piglets of the litter at rate $\beta I / N$ per day, with $I / N$ being the prevalence within the litter (number of infected piglets divided by litter size). In this formulation, $\beta$ is the transmission rate parameter, defined as the number of new infections caused by one infectious pig per day in a susceptible pen. As described in more detail by van Bunnik et al. (2012), it is possible to formulate the above model in terms of differential equations for the probabilities $q_{\left[S_{i}, N_{i}\right]}(t)$ to have $I_{i}$ infected pigs at time $t$, given that the pen started with $S_{i}$ susceptible pigs and one infected sow. These state probabilities (or "Master Equations") can be solved analytically (van Bunnik et al., 2012), to obtain explicit expressions for the probabilities $q_{\left[S_{i}, N_{i}\right]}\left(t_{i}\right)$ of observing $I_{i}$ cases at weaning age $t_{i}$, as in the data. These are combined in the log-likelihood function

$l_{2}\left(\boldsymbol{\lambda}_{\text {sow }}, \boldsymbol{\beta}\right)=\sum \log q_{\left[S_{i}, N_{i}\right]}\left(t_{i}\right)$

This equation was maximised to obtain the parameter estimates in Mathematica 8.0 (Wolfram Research, 2010). Four different models were fitted, with equal or different values for $\lambda_{\text {sow }}$ and $\beta$ in the four compartments, and they were compared to the beta-binomial models by means of AIC. Confidence intervals for the final model were obtained by profile-likelihood.

With the exception of the transmission analyses all statistical analyses were performed with $R$, version 2.11.1 ( $R$ Development Core Team, 2010).

\section{Results}

\subsection{Sow sample qPCR results}

All 76 selected sows were positive in qPCR analysis and the average amount of $A$. pleuropneumoniae CFUs in samples from farm A were significantly higher (median CFUs $=10^{6.4}$ (inter quartile range (IQR) $10^{5.9} ; 10^{6.7}$ )), than in samples from farm B (median CFUs $=10^{5.0}$ (IQR 10 ${ }^{4.3}$; $\left.\left.10^{5.9}\right)\right)(P<0.001)$ (Supplementary Table 2$)$. The qPCR sample results did not differ significantly between batches within farms $(P=0.14)$ and the qPCR results were not associated with parity $\left(r_{s p}=-0.19, P=0.11\right)$.

\subsection{Sow sample serology results}

All 76 serum samples from the sows tested positive in all antibody assays, with the exception of one sample from 
two sows from farm $B$, which gave inconclusive results in the ApxII antibody assay. These two sows and their litters were omitted from further analyses. The titres for ApxI antibodies were significantly higher in farm $A$ than in farm B $(P<0.001)$. For ApxII $(P<0.01)$ and Omp $(P<0.05)$, antibody titres were higher in farm B (Supplementary Table 2). Titres for ApxII antibodies were positively correlated with parity $\left(r_{s p}=0.37, P=0.001\right)$, whereas ApxIII and Omp antibody titres were negatively correlated with parity $\left(r_{s p}=-0.54\right.$ and $r_{s p}=-0.44$ respectively, $\left.P<0.001\right)$. ApxIII antibody titres were highly correlated to Omp antibody titres $\left(r_{s p}=0.80, P<0.001\right)$. Only ApxI antibody titres were correlated with qPCR results $\left(r_{s p}=0.34, P<0.01\right)$.

\subsection{Piglets results}

Litters were sampled at a median age of 24 days (range 15-30, IQR 23-25). The median size of litters at time of sampling was 12 (range 7-15 piglets), which was not related to parity. No possibly A. pleuropneumoniae related clinical signs or mortality were observed.

At the time of sampling 45 of 76 (0.59) litters had at least one positive piglet (Table 1 and Supplementary figure 1); this proportion did not differ significantly between farms or batches. In total, 238 of 871 piglets (0.27) tested positive (Table 1 ). The distribution of the proportions of positively tested piglets within litters showed a much higher variation (e.g. more litters without colonised piglets) than was expected based on a binomial distribution (Fig. 1). This is why we fitted models allowing description of variation by two mechanisms: the probability for a sow not to transmit the infection at all (the zero-inflation), and a beta-binomial distribution for the sows that do transmit the infection.

\subsection{Statistical model evaluation}

Table 2 shows the models with lowest AIC within each of the six subgroups. Across the six subgroups, the models with a beta-binomial distribution for the probability of infection within litters fitted much better (lower AIC) than those with a binomial distribution. Among those, the subgroup model without zero inflation (subgroup 2) fitted the data best (least complex model). Across all models, the simplest of subgroup 2, without dam characteristic explanatory variables, was considered to fit the data best. Inclusion of zero inflation into the final model of subgroup 2 did not improve the fit (two points higher in AIC), whereas adding any of the dam characteristic explanatory variables increased the AIC by $0-2$ points (not shown). From the best fitting model it was concluded that the probability of infection from a piglet is best described by a beta binomial distribution with $p_{\text {piglet }}=0.28(95 \% \mathrm{CI}: 0.21 ; 0.36)$ and $\rho=0.53$ (95\% CI: 0.42; 0.63).

The results of the power analysis showed that, with this study design, sample size and with the observed $p_{\text {piglet }}=0.28$ and variation $(\rho=0.53)$ a true difference in proportions of infected pigs of 0.20 can be detected with a power of $80 \%$.

Finally, the fit of a transmission model that also accounted for transmission amongst piglets ( $\mathrm{AIC}=316.5$ ) was less than the fit of a logistic regression model with a beta binomial distribution.

\section{Discussion}

The aim of this study was to investigate the pattern of clustering of colonisation of piglets with A. pleuropneumoniae at the end of the suckling period and to examine possible factors associated with the probability of colonisation.

It was shown, that colonisation of piglets with A. pleuropneumoniae was clustered across litters as well as within litters. Approximately $40 \%$ of the litters remained free from A. pleuropneumoniae during the suckling period, despite the finding that all sows tested positive for A. pleuropneumoniae, but no associated risk factors was identified that might explain this observation. Our findings are in accordance with the results of Vigre et al. (2002), as in both studies similar percentages of piglets tested positive before weaning, but in our study more information on the level of clustering on litter level before weaning was provided.

The best fit of a beta binomial model suggests some kind of random effect on litter level, but in this study no dam related factors could be identified that were associated with the probability of piglet infection. This may be explained if colonisation was not inhibited by the maternally derived antibodies that were investigated (anti-Apx I, ApxII, ApxIII and Omp antibodies), but rather by antibodies against other antigens or by other immunological, e.g. more cell mediated, factors. Indeed previous studies have suggested that anti-Apx and anti-Omp antibodies induced by vaccination are less likely to be associated with the susceptibility for colonisation (Velthuis et al., 2003), than with a protective effect against the development of clinical disease (Haga et al., 1997; Tumamao et al., 2004). It was anticipated that a possible association with dam parity would suggest such an alternative protective mechanism, as the level of immunity in sows is related to parity, either by antibodies (Klobasa and Butler, 1987), by interferon gamma (Uddin et al., 2010), or other mechanisms. For example, Miller et al. (2013) showed recently that although levels of immunity in sows were equal, parity effects on immunity were observed in offspring. In our study however, dam parity was not found to be associated to the infection probability of piglets. If there are any dam related risk factors for piglet colonisation by A. pleuropneumoniae, it is presently unclear what they are.

In positively tested litters also clustering was observed, as there were a few litters with very high proportions of positive litters. The poorer fit of the model that accounted for transmission within the litter compared to the fit of the beta binomial logistic regression model suggests that transmission among suckling piglets did not occur on a large scale. Nevertheless we cannot conclude that transmission among suckling piglets does not occur at all, as the study was not designed for this specific purpose.

The final models suggest that the infectiousness of the sows follows a beta distribution. Although the amount of A. pleuropneumoniae CFUs on the sow's tonsil was included 
Table 1

Numbers of positive and total tested litters and pigs for A. pleuropneumoniae colonisation in each cohort.

\begin{tabular}{|c|c|c|c|c|c|}
\hline & \multicolumn{2}{|l|}{ Farm A } & \multicolumn{2}{|l|}{ Farm B } & \multirow[t]{2}{*}{ Total } \\
\hline & Cohort 1 & Cohort 2 & Cohort 3 & Cohort 4 & \\
\hline Total pens tested & 18 & 18 & 20 & 20 & 76 \\
\hline Positively tested pens & 12 & 11 & 13 & 9 & 45 \\
\hline Proportion positive pens & 0.67 & 0.61 & 0.65 & 0.45 & 0.59 \\
\hline Total pigs tested & 193 & 199 & 238 & 241 & 871 \\
\hline Positively tested pigs & 54 & 73 & 70 & 41 & 238 \\
\hline Proportion positive pigs & 0.28 & 0.37 & 0.29 & 0.17 & 0.27 \\
\hline Mean proportion of positively tested piglets within positively tested litters & 0.43 & 0.60 & 0.45 & 0.37 & 0.46 \\
\hline
\end{tabular}

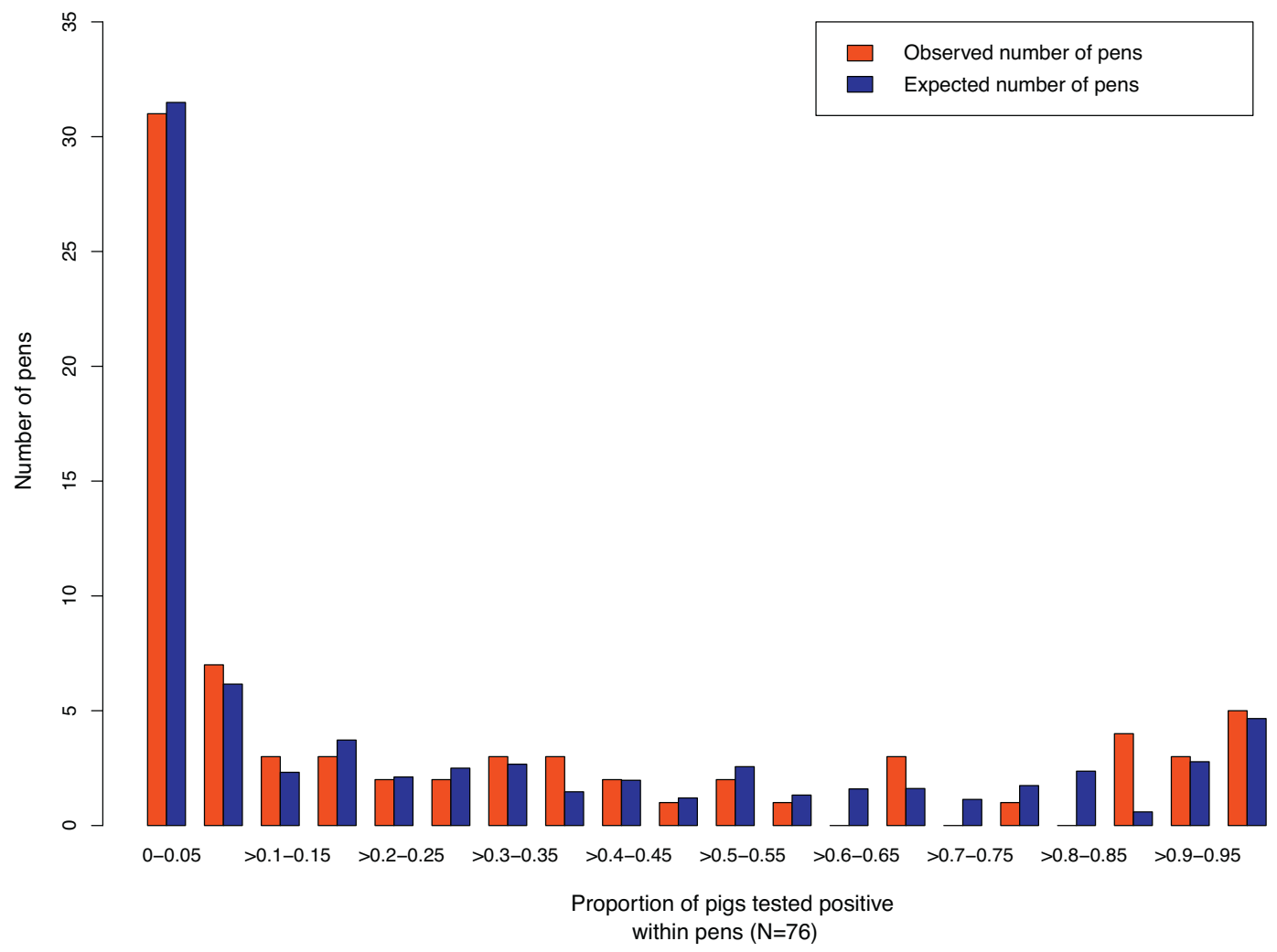

Fig. 1. Observed and expected frequencies of within pen prevalence. Expected values are calculated based given litter sizes and parameters obtained from the final model (beta binomial probability distribution with $p_{\text {piglet }}=0.28$ and $\rho=0.53$ ).

Table 2

Results of model selection procedure.

\begin{tabular}{|c|c|c|c|c|c|}
\hline \multirow[t]{2}{*}{$\begin{array}{l}\text { Model } \\
\text { Sub-group }\end{array}$} & \multirow[t]{2}{*}{ Zero inflation } & \multicolumn{2}{|c|}{$\begin{array}{l}\text { Inclusion of dam characteristic } \\
\text { explanatory variables in }\end{array}$} & \multirow[t]{2}{*}{ Probability distribution } & \multirow[t]{2}{*}{ AIC } \\
\hline & & $p_{d a m}$ & $p_{\text {piglet }}$ & & \\
\hline 1 & - & - & $\mathrm{X}$ & Binomial & 610.0 \\
\hline 2 & - & - & $\mathbf{X}$ & Beta binomial & 308.0 \\
\hline 3 & $\mathrm{X}$ & $\mathrm{X}$ & - & Binomial & 453.0 \\
\hline 4 & $\mathrm{X}$ & $\mathrm{X}$ & - & Beta binomial & 310.0 \\
\hline 5 & $\mathrm{X}$ & - & $\mathrm{X}$ & Binomial & 434.7 \\
\hline 6 & $\mathrm{X}$ & - & $\mathrm{X}$ & Beta binomial & 310.0 \\
\hline 7 & $\mathrm{X}$ & $\mathrm{X}$ & $X$ & Binomial & 443.9 \\
\hline 8 & $\mathrm{X}$ & $\mathrm{X}$ & $\mathrm{X}$ & Beta binomial & 309.9 \\
\hline
\end{tabular}

For each subgroup of models the model with lowest AIC is reported. The subgroup model with lowest AIC is printed in bold. $\mathrm{X}=$ parameter included. 
to account for infectiousness, no association was found with colonisation in offspring. This may be explained by the fact that sows were sampled only once, three weeks before parturition, and the infectivity of individual pigs has shown to vary considerably from day to day (Velthuis et al., 2002).

There was more variation in colonised piglets per litter than expected. It is unclear whether these results were found because a strict hygiene and animal management protocol was applied, or whether transmission between pens would not occur on a large scale anyway. For extrapolation of the results to the field, the unexplained large variation in colonised piglets per litter may be the main obstacle, as in regular pig farming these preventive measures are applied less strictly.

Results of this study do not indicate towards a specific point of control at dam level to reduce the proportion of colonised piglets at weaning. However, as many litters tested negative, the results of this study suggest that disease control measures in the farrowing room in general should aim to prevent transmission between litters to minimise the number of infected litters. It remains, however, uncertain if litters free from A. pleuropneumoniae remain free after weaning, or if they become colonised quickly after movement to weaner or finisher units. A follow-up study could be performed to evaluate subsequent transmission and colonisation after weaning.

\section{Conclusion}

The colonisation status of piglets for A. pleuropneumoniae was found to be clustered across and within litters, even though all sows were tested positive for A. pleuropneumoniae before farrowing. No dam related variables were found to be associated to either the probability of a litter to become infected, or the probability of individual pigs to become infected.

\section{Acknowledgements}

The farmers and the farm workers are greatly acknowledged for their hospitality and execution of the research protocol. MSD Animal Health, Boxmeer, the Netherlands is acknowledged for analysis of the serum samples. MSD was not involved in design of the study, data analysis or in composing the manuscript. The Central Veterinary Institute is acknowledged for serovar determination of the A. pleuropneumoniae isolates. Manon Houben, Wouter van Herten and Piet Dirven, are acknowledged for their cooperation. Katrien van Dongen, Bram Goesten, Leonie de Louw and all volunteers are acknowledged for assistance with sampling and data collection. Finally, anonymous reviewers are greatly acknowledged for their advice, resulting in significant improvements to the manuscript.

\section{Appendix A. Supplementary data}

Supplementary data associated with this article can be found, in the online version, at http://dx.doi.org/10.1016/j.prevetmed.2014.02.008.

\section{References}

Agresti, A., 2013. Categorical Data Analysis. John Wiley \& Sons, Hoboken, NJ, United States of America.

Angen, O., Andreasen, M., Nielsen, E.O., Stockmarr, A., Baekbo, P., 2008. Effect of tulathromycin on the carrier status of Actinobacillus pleuropneumoniae serotype 2 in the tonsils of pigs. Vet. Rec. 163, 445-447.

Burnham, K.P., Anderson, D.R., 2002. Model Selection and Multimodel Inference, A Practical Information-Theoretic Approach. Springer, New York, USA.

van Bunnik, B.A., Katsma, W.E., Wagenaar, J.A., Jacobs-Reitsma, W.F., de Jong, M.C., 2012. Acidification of drinking water inhibits indirect transmission but not direct transmission of campylobacter between broilers. Prev. Vet. Med. 105, 315-319.

Cheung, Y.B., 2006. Growth and cognitive function of Indonesian children: zero-inflated proportion models. Stat. Med. 25, 3011-3022.

Chiers, K., Donne, E., Van Overbeke, I., Ducatelle, R., Haesebrouck, F., 2002. Actinobacillus pleuropneumoniae infections in closed swine herds: infection patterns and serological profiles. Vet. Microbiol. 85, 343-352.

Cruijsen, T., van Leengoed, L.A., Kamp, E.M., Bartelse, A., Korevaar, A., Verheijden, J.H., 1995a. Susceptibility to Actinobacillus pleuropneumoniae infection in pigs from an endemically infected herd is related to the presence of toxin-neutralizing antibodies. Vet. Microbiol. 47, 219-228.

Cruijsen, T., van Leengoed, L.A., Kamp, E.M., Hunneman, W.A., Riepema, K., Bartelse, A., Verheijden, J.H., 1995b. Prevalence and development of antibodies neutralizing the haemolysin and cytotoxin of Actinobacillus pleuropneumoniae in three infected pig herds. Vet. Q. 17, 96-100.

Fablet, C., Marois, C., Kuntz-Simon, G., Rose, N., Dorenlor, V., Eono, F., Eveno, E., Jolly, J.P., Le Devendec, L., Tocqueville, V., Quéguiner, S., Gorin, S., Kobisch, M., Madec, F., 2011. Longitudinal study of respiratory infection patterns of breeding sows in five farrow-to-finish herds. Vet. Microbiol. 147, 329-339.

Gottschalk, M., Taylor, D.J., 2006. Actinobacillus pleuropneumoniae. In: Straw, B.E., Zimmerman, J.J., D’Allaire, S., Taylor, D.J. (Eds.), Diseases of Swine. Blackwell Publishing, Ames, IA, USA, pp. 563-576.

Haga, Y., Ogino, S., Ohashi, S., Ajito, T., Hashimoto, K., Sawada, T., 1997. Protective efficacy of an affinity-purified hemolysin vaccine against experimental swine pleuropneumonia. J. Vet. Med. Sci. 59, 115-120.

Hamer-Barrera, R., Godinez, D., Enriquez, V.I., Vaca-Pacheco, S., MartinezZuniga, R., Talamas-Rohana, P., Suarez-Guemez, F., de la Garza, M., 2004. Adherence of Actinobacillus pleuropneumoniae serotype 1 to swine buccal epithelial cells involves fibronectin. Can. J. Vet. Res. 68, 33-41.

Hoflack, G., Maes, D., Mateusen, B., Verdonck, M., de Kruif, A., 2001. Efficacy of tilmicosin phosphate (pulmotil premix) in feed for the treatment of a clinical outbreak of Actinobacillus pleuropneumoniae infection in growing-finishing pigs. J. Vet. Med. B: Infect. Dis. Vet. Public Health 48, 655-664.

Klobasa, F., Butler, J.E., 1987. Absolute and relative concentrations of immunoglobulins $\mathrm{G}, \mathrm{M}$, and $\mathrm{A}$, and albumin in the lacteal secretion of sows of different lactation numbers. Am. J. Vet. Res. 48, 176-182.

Kobisch, M., van den Bosch, J.F., 1992. Efficacy of an Actinobacillus pleuropneumoniae subunit vaccine. In: Proceedings of the 12th International Pig Veterinary Society Congress, The Hague, The Netherlands, p. 216.

Mengelers, M.J., Kuiper, H.A., Pijpers, A., Verheijden, J.H., van Miert, A.S., 2000. Prevention of pleuropneumonia in pigs by in-feed medication with sulphadimethoxine and sulphamethoxazole in combination with trimethoprim. Vet. Q. 22, 157-162.

Miller, Y.J., Collins, A.M., Emery, D., Begg, D.J., Smits, R.J., Holyoake, P.K., 2013. Piglet performance and immunity is determined by the parity of both the birth dam and the rearing dam. Anim. Prod. Sci. 53, 46-51.

Nielsen, R., van den Bosch, J.F., Plambeck, T., Sorensen, V., Nielsen, J.P., 2000. Evaluation of an indirect enzyme-linked immunosorbent assay (ELISA) for detection of antibodies to the apx toxins of Actinobacillus pleuropneumoniae. Vet. Microbiol. 71, 81-87.

R Development Core Team, 2010. R: A Language and Environment for Statistical Computing 2.11.1. R Foundation for Statistical Computing, Vienna, Austria http://www.R-project.org

Shin, M.K., Kang, M.L., Cha, S.B., Lee, W.J., Sung, J.H., Yoo, H.S., 2011. An immunosorbent assay based on the recombinant Apxla, ApxIla, and ApxIIIa toxins of Actinobacillus pleuropneumoniae and its application to field sera. J. Vet. Diagn. Invest. 23, 736-742.

Sjölund, M., Zoric, M., Persson, M., Karlsson, G., Wallgren, P., 2011. Disease patterns and immune responses in the offspring to sows with high or low antibody levels to Actinobacillus pleuropneumoniae serotype 2. Res. Vet. Sci. 91, 25-31. 
Tobias, T.J., Bouma, A., Klinkenberg, D., Daemen, A.J.J.M., Stegeman, J.A., Wagenaar, J.A., Duim, B., 2012. Detection of Actinobacillus pleuropneumoniae in pigs by real-time quantitative PCR for the apxIVA gene. Vet. J. 193, 557-560.

Tumamao, J.Q., Bowles, R.E., Van Den Bosch, H., Klaasen, H.L.B.M., Fenwick, B.W., Storie, G.J., Blackall, P.J., 2004. Comparison of the efficacy of a subunit and a live streptomycin-dependent porcine pleuropneumonia vaccine. Aust. Vet. J. 82, 370-374.

Uddin, M.J., Grosse-Brinkhaus, C., Cinar, M.U., Jonas, E., Tesfaye, D., Tholen, E., Juengst, H., Looft, C., Ponsuksili, S., Wimmers, K., Phatsara, C., Schellander, K., 2010. Mapping of quantitative trait loci for mycoplasma and tetanus antibodies and interferon-gamma in a porcine F2 duroc $\times$ pietrain resource population. Mamm. Genome 21, 409-418.
Velthuis, A.G., De Jong, M.C., Stockhofe, N., Vermeulen, T.M., Kamp, E.M., 2002. Transmission of Actinobacillus pleuropneumoniae in pigs is characterized by variation in infectivity. Epidemiol. Infect 129, 203-214.

Velthuis, A.G., De Jong, M.C., Kamp, E.M., Stockhofe, N., Verheijden, J.H., 2003. Design and analysis of an Actinobacillus pleuropneumoniae transmission experiment. Prev. Vet. Med. 60, 53-68.

Vigre, H., Angen, O., Barfod, K., Lavritsen, D.T., Sorensen, V., 2002. Transmission of Actinobacillus pleuropneumoniae in pigs under field-like conditions Emphasis on tonsillar colonisation and passively acquired colostral antibodies. Vet. Microbiol. 89, $151-159$.

Wolfram Research, 2010. Mathematica 8.0, Champaign, IL, United States. http://www.wolfram.com 